Pacific

Journal of

Mathematics

\title{
TWO-DIMENSIONAL DISJOINT MINIMAL GRAPHS
}

\section{LINFENG ZHOU}




\title{
TWO-DIMENSIONAL DISJOINT MINIMAL GRAPHS
}

\author{
LINFENG ZHOU
}

\begin{abstract}
Under the assumption of Gauss curvature vanishing at infinity, we prove Meeks' conjecture: the number of disjointly supported minimal graphs in $\mathbb{R}^{3}$ is at most two.
\end{abstract}

\section{Introduction}

Let $\Omega$ be an open subset in $\mathbb{R}^{2}$ and denote its boundary by $\partial \Omega$. As we know, if a function $u(x)$ defined on $\Omega$ satisfies the equation

$$
\operatorname{div} \frac{\nabla u}{\sqrt{1+|\nabla u|^{2}}}=0
$$

$G=\{(x, u(x)): x \in \Omega\}$ is called a minimal graph in $\mathbb{R}^{3}$. We say the minimal graph $G$ is supported on $\Omega$ if $\left.u\right|_{\partial \Omega}=0$ and $u \geq 0$.

Meeks [2005] has conjectured that the number of disjointly supported minimal graphs with zero boundary values over an open subset in $\mathbb{R}^{2}$ is at most 2 . In fact, for arbitrary dimension, Meeks and Rosenberg [2005] proved if a set of disjointly supported minimal graphs have bounded gradient, then the number of the graphs must be finite. Later, Li and Wang [2001] gave an upper bound of the number of the graphs without any assumption on the growth rate of each graph. As a corollary, when minimal graphs are two dimensional in $\mathbb{R}^{3}$, they obtained the number is at most 24. At the same time, Spruck [2002] proved that there are at most two admissible sublinear growth solution pairs of Equation (1) defined over disjoint domains. Recently, by using angular density, Tkachev [2009] showed the number of two dimensional disjointly supported minimal graphs is less than or equals 3 .

Observing the similarity between disjoint $d$-massive sets and disjointly supported minimal graphs, we can apply the method for proving the finiteness theorem of disjoint $d$-massive sets in $\mathbb{R}^{2}$ [Li and Wang 1999] to study disjoint minimal graphs. We obtain the following theorem:

MSC2010: 53C42, 53C21.

Keywords: minimal graphs, Meeks conjecture. 
Theorem 1.1. Suppose $\left\{G_{i}=\left(\Omega_{i}, u_{i}\right)\right\}_{i=1}^{k}$ is a set of disjointly supported minimal graphs in $\mathbb{R}^{3}$, where each $\Omega_{i}$ is an open subset in $\mathbb{R}^{2}$. If the Gauss curvature $K_{i}(x)$ of each graph satisfies

$$
K_{i}(x) \rightarrow 0 \quad \text { as }|x| \rightarrow \infty,
$$

then the number $k$ is at most two.

By choosing a different region of integration, one obtains an improvement on a theorem of Spruck [2002]:

Corollary 1.2. Suppose $\left\{G_{i}=\left(\Omega_{i}, u_{i}\right)\right\}_{i=1}^{k}$ is a set of disjointly supported minimal graphs in $\mathbb{R}^{3}$, where each $\Omega_{i}$ is an open subset in $\mathbb{R}^{2}$. If each graph has sublinear growth, then $k$ is at most two.

\section{Proof of Theorem 1.1}

We denote the 3-dimensional ball of radius $R$ centered at the origin of $\mathbb{R}^{3}$ by $B^{3}(R)$ and the 2-dimensional sphere of radius $R$ by $S^{2}(R)$. The key is to estimate the sum of all curves' length $\ell\left(G_{i} \cap S^{2}(R)\right)$ when $R$ is sufficiently large.

Theorem 2.1. Suppose $\left\{G_{i}=\left(\Omega_{i}, u_{i}\right)\right\}_{i=1}^{k}$ is a set of disjointly supported minimal graphs in $\mathbb{R}^{3}$, where the Gauss curvature $K_{i}(x)$ of each $G_{i}$ satisfies

$$
K_{i}(x) \rightarrow 0 \text { as }|x| \rightarrow \infty .
$$

For a sufficiently large radius $R$, we have the bound

$$
\sum_{i=1}^{k} \ell\left(G_{i} \cap S^{2}(R)\right) \leq \pi^{2} R+o(1) R .
$$

In the particular case when $k=3$, we have the better estimate

$$
\sum_{i=1}^{3} \ell\left(G_{i} \cap S^{2}(R)\right) \leq 2 \sqrt{2} \pi R+o(1) R .
$$

Before proving this, we introduce a lemma.

Lemma 2.2. Let $B_{+}^{3}(R)$ be a 3-dimensional upper half-ball with radius $R$ and let $S_{+}^{2}(R)$ be a 2-dimensional upper half-sphere. Suppose $\pi_{i}: G_{i} \rightarrow \mathbb{R}^{2}$ is the natural projection map. If $\Sigma_{1}, \Sigma_{2}, \ldots, \Sigma_{s}$ are planes in $\mathbb{R}^{3}$ such that the interiors of $\pi_{i}\left(\Sigma_{i} \cap B_{+}^{3}(R)\right)$ are pairwise disjoint for sufficiently large $R$, we have

$$
\sum_{i=1}^{s} \ell\left(\Sigma_{i} \cap S_{+}^{2}(R)\right) \leq \pi^{2} R
$$

Moreover, when $s=3$, we have the better estimate

$$
\sum_{i=1}^{3} \ell\left(\Sigma_{i} \cap S_{+}^{2}(R)\right) \leq 2 \sqrt{2} \pi R
$$


Proof. Suppose $D(R)=\left\{\left(x_{1}, x_{1}, 0\right): x_{1}^{2}+x_{2}^{2} \leq R^{2}\right\}$ is a disk in $\mathbb{R}^{3}$. Since each $\Sigma_{i}$ is a plane, $\Sigma_{i} \cap D(R)$ is a chord; let $\theta_{i}$ be the corresponding central angle. Here we only need to consider the case that the union $\bigcup_{i=1}^{s}\left(\Sigma_{i} \cap D(R)\right)$ is a polygon; otherwise, one can add more planes still satisfying the required conditions and such that the union of chords becomes a polygon.

If the center of the disk $D(R)$ is in the interior of the polygon or on one of the edges of the polygon, each central angle $\theta_{i}$ satisfies $0<\theta_{i} \leq \pi$. Since the interiors of the $\pi_{i}\left(\Sigma_{i} \cap B_{+}^{3}(R)\right)$ are pairwise disjoint, a simple computation yields the bound

$$
\ell\left(\Sigma_{i} \cap S_{+}^{2}(R)\right) \leq \pi R \sin \frac{\theta_{i}}{2}
$$

on the length of the $\operatorname{arc} \ell\left(\Sigma_{i} \cap S_{+}^{2}(R)\right)$. The right-hand side achieves the maximum if and only if $\Sigma_{i}$ is perpendicular to the disk $D(R)$. Thus

$$
\begin{aligned}
\sum_{i=1}^{s} \ell\left(\Sigma_{i} \cap S_{+}^{2}(R)\right) & \leq \sum_{i=1}^{s} \pi R \sin \frac{\theta_{i}}{2} \leq \pi R s \sin \left(\frac{1}{s} \sum_{i}^{s} \frac{\theta_{i}}{2}\right) \\
& \leq \pi R s \sin \frac{\pi}{s} \leq \pi^{2} R .
\end{aligned}
$$

In the second inequality, we have used the concave property of the sine function on the interval $[0, \pi]$.

For the special case when $s=3$, one gets from (2)

$$
\sum_{i=1}^{3} \ell\left(\Sigma_{i} \cap S_{+}^{2}(R)\right) \leq 3 \pi R \sin \frac{\pi}{3}=\frac{3 \sqrt{3}}{2} \pi R .
$$

If the center of the disk $D(R)$ is outside the polygon, there exists an $i_{0}$ such that $\theta_{i_{0}}>\pi$. For simplicity, let us assume $i_{0}=s$. A similar computation leads to

$$
\ell\left(\Sigma_{i} \cap S_{+}^{2}(R)\right) \leq \begin{cases}\pi R \sin \frac{\theta_{i}}{2} & \text { for } 1 \leq i \leq s-1, \\ R \theta_{s} & \text { for } i=s .\end{cases}
$$

In the first case, equality holds if and only if $\Sigma_{i}$ is perpendicular to the disk, and in the second, if and only if $\Sigma_{s}$ is in the same plane of the disk $D(R)$. Hence

$$
\begin{aligned}
\sum_{i=1}^{s} \ell\left(\Sigma_{i} \cap S_{+}^{2}(R)\right) & \leq \sum_{i=1}^{s-1} \pi R \sin \frac{\theta_{i}}{2}+R \theta_{s} \leq \sum_{i=1}^{s-1} \pi R \sin \frac{\theta_{i}}{2}+2 \pi R \sin \frac{\theta_{s}}{4} \\
& \leq \pi R(s+1) \sin \frac{\pi}{s+1} \leq \pi^{2} R .
\end{aligned}
$$


If $s=3$, by (4) we obtain that

$$
\sum_{i=1}^{3} \ell\left(\Sigma_{i} \cap S_{+}^{2}(R)\right) \leq 4 \pi R \sin \frac{\pi}{4}=2 \sqrt{2} \pi R
$$

The conclusion is derived from (2), (4) and (3), (5).

Proof of Theorem 2.1. For each minimal graph $G_{i}$, since the Gauss curvature $K_{i}=0$ at infinity, it means $G_{i}$ is asymptotic to a flat plane. Therefore, we can use the intersection of a plane $\Sigma_{i}$ and $S_{+}^{2}(R)$ to approximate the curve $G_{i} \cap S^{2}(R)$. By Lemma 2.2, one has

$$
\ell\left(G_{i} \cap S^{2}(R)\right) \leq \ell\left(\Sigma_{i} \cap S_{+}^{2}(R)\right)+o(1) R .
$$

Therefore

$$
\sum_{i=1}^{k} \ell\left(G_{i} \cap S^{2}(R)\right) \leq \sum_{i=1}^{k} \ell\left(\Sigma_{i} \cap S_{+}^{2}(R)\right)+o(1) R \leq \pi^{2} R+0(1) R .
$$

The following area growth estimate of a minimal graph is proved using a wellknown argument; one can see [Li and Wang 2001] for the details.

Lemma 2.3. If $G=(\Omega, u)$ is a minimal graph in $\mathbb{R}^{3}$, the area of $G \cap B^{3}(R)$ satisfies

$$
A\left(G \cap B^{3}(R)\right) \leq 3 \pi R^{2} .
$$

Proof of Theorem 1.1. Let $B^{3}(R)$ be the ball of radius $R$ in $\mathbb{R}^{3}$. Since

$$
\int_{G_{i} \cap B^{3}(R)}\left|\tilde{\nabla} u_{i}\right|^{2} \leq \int_{\left.G_{i} \cap \partial B^{3}(R)\right)} u_{i}\left(\tilde{\nabla} u_{i} \cdot \frac{\partial}{\partial r}\right),
$$

where $\tilde{\nabla}$ means the gradient operator on $G_{i}$, one has

$$
\begin{aligned}
2 \lambda_{1}^{1 / 2}\left(G_{i} \cap \partial B^{3}(R)\right) & \int_{G_{i} \cap B^{3}(R)}\left|\tilde{\nabla} u_{i}\right|^{2} \\
& \leq 2 \lambda_{1}^{1 / 2} \int_{G_{i} \cap \partial B^{3}(R)} u_{i} \cdot \frac{\partial u_{i}}{\partial r} \\
& \leq \lambda_{1} \int_{G_{i} \cap \partial B^{3}(R)} u_{i}^{2}+\int_{G_{i} \cap \partial B^{3}(R)}\left(\frac{\partial u_{i}}{\partial r}\right)^{2} \\
& \leq \int_{G_{i} \cap \partial B^{3}(R)}\left|\bar{\nabla} u_{i}\right|^{2}+\int_{G_{i} \cap \partial B^{3}(R)}\left(\frac{\partial u_{i}}{\partial r}\right)^{2}=\int_{G_{i} \cap \partial B^{3}(R)}\left|\tilde{\nabla} u_{i}\right|^{2} .
\end{aligned}
$$

Here $\lambda_{1}^{1 / 2}\left(G_{i} \cap \partial B^{3}(R)\right)$ denotes the first Dirichlet eigenvalue on $G_{i} \cap \partial B^{3}(R)$. We know that

$$
\lambda_{1}^{1 / 2}\left(G_{i} \cap \partial B^{3}(R)\right) \geq \frac{\pi^{2}}{\ell^{2}\left(G_{i} \cap \partial B^{3}(R)\right)}
$$


in $\mathbb{R}^{3}$. Therefore

$$
\frac{\int_{G_{i} \cap \partial B^{3}(R)}\left|\tilde{\nabla} u_{i}\right|^{2}}{\int_{G_{i} \cap B^{3}(R)}\left|\tilde{\nabla} u_{i}\right|^{2}} \geq 2 \lambda_{1}^{1 / 2} \geq \frac{2 \pi}{\ell\left(\Gamma_{i}\right)},
$$

where $\Gamma_{i}:=G_{i} \cap \partial B^{3}(R)$. Thus we obtain

$$
\sum_{i=1}^{k} \frac{\int_{G_{i} \cap \partial B^{3}(R)}\left|\tilde{\nabla} u_{i}\right|^{2}}{\int_{G_{i} \cap B^{3}(R)}\left|\tilde{\nabla} u_{i}\right|^{2}} \geq \sum_{i=1}^{k} \frac{2 \pi}{\ell\left(\Gamma_{i}\right)} .
$$

Notice that

$$
k^{2} \leq\left(\sum_{i=1}^{k} \ell\left(\Gamma_{i}\right)\right)\left(\sum_{i=1}^{k} \frac{1}{\ell\left(\Gamma_{i}\right)}\right) .
$$

According to Theorem 2.1, one has

$$
\sum_{i=1}^{k} \ell\left(\Gamma_{i}\right) \leq \pi^{2} R+o(1) R
$$

for a sufficiently large radius $R$. Then it can be concluded that

$$
\sum_{i=1}^{k} \frac{\int_{G_{i} \cap \partial B^{3}(R)}\left|\tilde{\nabla} u_{i}\right|^{2}}{\int_{G_{i} \cap B^{3}(R)}\left|\tilde{\nabla} u_{i}\right|^{2}} \geq \frac{2 \pi k^{2}}{R\left(\pi^{2}+o(1)\right)} .
$$

Observing that

$$
\int_{G_{i} \cap \partial B^{3}(r)}\left|\tilde{\nabla} u_{i}\right|^{2}=\frac{\partial}{\partial r} \int_{G_{i} \cap B^{3}(r)}\left|\tilde{\nabla} u_{i}\right|^{2},
$$

we obtain from (6) that

$$
\ln \prod_{i=1}^{k} \frac{\int_{G_{i} \cap B^{3}(R)}\left|\tilde{\nabla} u_{i}\right|^{2}}{\int_{G_{i} \cap B^{3}\left(R_{0}\right)}\left|\tilde{\nabla} u_{i}\right|^{2}} \geq \frac{2 \pi k^{2}}{\pi^{2}+o(1)} \ln \frac{R}{R_{0}} .
$$

Let $\left(x, y, u_{i}(x, y)\right)$ be a parametrization of $G_{i}$, so the induced metric on $G_{i}$ is

$$
d s^{2}=\left(1+\left(u_{i}\right)_{x}^{2}\right) d x^{2}+2\left(u_{i}\right)_{x}\left(u_{i}\right)_{y} d x d y+\left(1+\left(u_{i}\right)_{y}^{2}\right) d y^{2} .
$$

We then have

$$
\left|\tilde{\nabla} u_{i}\right|=\sqrt{u_{x^{i}} u_{x^{j}} g^{i j}}=\sqrt{\frac{\left|\nabla u_{i}\right|^{2}}{1+\left|\nabla u_{i}\right|^{2}}} \leq 1,
$$

from which one can deduce

$$
\prod_{i=1}^{k} \int_{\left.G_{i} \cap B^{3}(R)\right)}\left|\tilde{\nabla} u_{i}\right|^{2} \leq A^{k}\left(G_{i} \cap B^{3}(R)\right) \leq\left(3 \pi R^{2}\right)^{k} .
$$


Combining (8) and (9) implies

$$
\frac{2 \pi k^{2}}{\pi^{2}+o(1)}\left(\ln R-\ln R_{0}\right) \leq 2 k \ln R+c_{1} .
$$

Letting $R \rightarrow+\infty$ we see that $k \leq \pi$; in particular, $k \leq 3$.

If $k=3$, an analogous argument using the refined length estimate in Theorem 2.1 leads to $k \leq 2 \sqrt{2}$, which is a contradiction. Thus $k$ has to be at most 2 .

Remark. Tkachev [2009] has already proved the number of two dimensional disjointly supported minimal graphs is at most 3 . Here a different approach can lead to a better estimate if assuming the Gauss curvature vanishes at infinity.

\section{Proof of Corollary 1.2}

Let $\pi_{i}: G_{i} \rightarrow \mathbb{R}^{2}$ be the natural projective map and $B^{2}(R)$ be the ball of radius $R$ in $\mathbb{R}^{2}$. By employing the same method in the proof of Theorem 1.1 except for using a different region of integration $\pi_{i}^{-1}\left(\Omega_{i} \cap B^{2}(R)\right)$, one can conclude

Theorem 3.1. Suppose $\left\{\left(\Omega_{i}, u_{i}\right)\right\}_{i=1}^{k}$ is a set of disjointly supported minimal graphs in $R^{3}$ where each $\Omega_{i}$ is an open subset in $R^{2}$. If the gradient of each $u_{i}$ is bounded, say $\left|\nabla u_{i}\right| \leq c$, then $k$ satisfies $k \leq 2 \sqrt{1+c^{2}}$.

Proof. By a similar argument, one can obtain that

$$
\sum_{i=1}^{k} \frac{\int_{\pi_{i}^{-1}\left(\Omega_{i} \cap \partial B^{2}(R)\right)}\left|\tilde{\nabla} u_{i}\right|^{2}}{\int_{\pi_{i}^{-1}\left(\Omega_{i} \cap B^{2}(R)\right)}\left|\tilde{\nabla} u_{i}\right|^{2}} \geq \frac{2 \pi k^{2}}{\sum_{i=1}^{k} \ell\left(\Gamma_{i}\right)} .
$$

where $\Gamma_{i}:=\pi_{i}^{-1}\left(\Omega_{i} \cap \partial B^{2}(R)\right)$. If one chooses the parametrization

$$
\left(R \cos \theta, R \sin \theta, u_{i}(R \cos \theta, R \sin \theta)\right)
$$

for the curve $\Gamma_{i}$ and assume $\left|\nabla u_{i}\right| \leq c$, then

$$
\begin{aligned}
\ell\left(\Gamma_{i}\right) & =\int_{\theta_{0}}^{\theta_{1}} \sqrt{R^{2}+\left(-\left(u_{i}\right)_{x} R \sin (\theta)+\left(u_{i}\right)_{y} R \cos (\theta)\right)^{2}} d \theta \\
& \leq \int_{\theta_{0}}^{\theta_{1}} \sqrt{R^{2}+\left(\left(u_{i}\right)_{x}^{2}+\left(u_{i}\right)_{y}^{2}\right)\left(R^{2} \sin (\theta)^{2}+R^{2} \cos (\theta)^{2}\right)} d \theta \\
& \leq\left(\theta_{1}-\theta_{0}\right) R \sqrt{1+c^{2}} .
\end{aligned}
$$

Since the minimal graphs are disjoint, we get

$$
\sum_{i=1}^{k} \ell\left(\Gamma_{i}\right) \leq 2 \pi R \sqrt{1+c^{2}} .
$$


Then it can be concluded that

$$
\sum_{i=1}^{k} \frac{\int_{\pi_{i}^{-1}\left(\Omega_{i} \cap \partial B^{2}(R)\right)}\left|\tilde{\nabla} u_{i}\right|^{2}}{\int_{\pi_{i}^{-1}\left(\Omega_{i} \cap B^{2}(R)\right)}\left|\tilde{\nabla} u_{i}\right|^{2}} \geq \frac{k^{2}}{R \sqrt{1+c^{2}}} .
$$

Integrating (10), one obtains

$$
\ln \prod_{i=1}^{k} \frac{\int_{\pi_{i}^{-1}\left(\Omega_{i} \cap \partial B^{2}(R)\right)}\left|\tilde{\nabla} u_{i}\right|^{2}}{\int_{\pi_{i}^{-1}\left(\Omega_{i} \cap B^{2}\left(R_{0}\right)\right)}\left|\tilde{\nabla} u_{i}\right|^{2}} \geq \frac{k^{2}}{\sqrt{1+c^{2}}} \ln \frac{R}{R_{0}} .
$$

On the other hand,

$$
\begin{aligned}
\prod_{i=1}^{k} \int_{\pi_{i}^{-1}\left(\Omega_{i} \cap B^{2}(R)\right)}\left|\tilde{\nabla} u_{i}\right|^{2} & \leq A^{k}\left(\pi_{i}^{-1}\left(\Omega_{i} \cap B^{2}(R)\right)\right) \\
& =\left(\int_{\Omega_{i} \cap B^{2}(R)} \sqrt{1+|\nabla u|^{2}}\right)^{k} \leq\left(\sqrt{1+c^{2}} \pi R^{2}\right)^{k} .
\end{aligned}
$$

Combining (11) and (12), we have

$$
\frac{k^{2}}{\sqrt{1+c^{2}}}\left(\ln R-\ln R_{0}\right) \leq 2 k \ln R+c_{1} .
$$

Letting $R \rightarrow+\infty$ yields

$$
k \leq 2 \sqrt{1+c^{2}} .
$$

Obviously, Corollary 1.2 follows from above theorem when each graph satisfies

$$
\left|\nabla u_{i}\right| \rightarrow 0 \quad(|x| \rightarrow+\infty) .
$$

Remark. J. Spruck [2002] proved Corollary 1.2 under the assumption of a certain decay rate at infinity for the Gauss curvature. However, here we do not need any restrictions on the Gauss curvature.

\section{Acknowledgements}

This work was carried out while the author was visiting the University of Minnesota. He would like to express his gratitude to Professor Jiaping Wang for his guidance and many helpful discussions. The author also thanks the university's School of Mathematics UMN for its hospitality, and the referees for many valuable comments.

\section{References}

[Li and Wang 1999] P. Li and J. Wang, "Counting massive sets and dimensions of harmonic functions”, J. Differential Geom. 53:2 (1999), 237-278. MR 2001k:53063 Zbl 1039.53036 
[Li and Wang 2001] P. Li and J. Wang, "Finiteness of disjoint minimal graphs", Math. Res. Lett. 8:6 (2001), 771-777. MR 2002k:53012 Zbl 0999.05055

[Meeks 2005] W. H. Meeks, III, "Global problems in classical minimal surface theory", pp. 453469 in Global theory of minimal surfaces (Berkeley, CA, 2001), edited by D. Hoffman, Clay Math. Proc. 2, Amer. Math. Soc., Providence, RI, 2005. MR 2006f:53011 Zbl 1100.53012

[Meeks and Rosenberg 2005] W. H. Meeks, III and H. Rosenberg, "The uniqueness of the helicoid", Ann. of Math. (2) 161:2 (2005), 727-758. MR 2006f:53012 Zbl 1102.53005

[Spruck 2002] J. Spruck, "Two-dimensional minimal graphs over unbounded domains", J. Inst. Math. Jussieu 1:4 (2002), 631-640. MR 2004e:53010 Zbl 1038.53012

[Tkachev 2009] V. G. Tkachev, "Disjoint minimal graphs", Ann. Global Anal. Geom. 35:2 (2009), 139-155. MR 2010c:53011 Zbl 1171.53012

Received June 27, 2011. Revised November 14, 2011.

LINFENG ZHOU

DEPARTMENT OF MATHEMATICS

EAST CHINA NORMAL UNIVERSITY

SHANGHAI 200241

CHINA

lfzhou@math.ecnu.edu.cn 


\title{
PACIFIC JOURNAL OF MATHEMATICS
}

\author{
http://pacificmath.org \\ Founded in 1951 by \\ E. F. Beckenbach (1906-1982) and F. Wolf (1904-1989)
}

\section{EDITORS}

V. S. Varadarajan (Managing Editor)

Department of Mathematics

University of California

Los Angeles, CA 90095-1555

pacific@math.ucla.edu

Vyjayanthi Chari

Department of Mathematics

University of California

Riverside, CA 92521-0135

chari@math.ucr.edu

\section{Robert Finn}

Department of Mathematics Stanford University

Stanford, CA 94305-2125

finn@math.stanford.edu

Kefeng Liu

Department of Mathematics

University of California

Los Angeles, CA 90095-1555

liu@math.ucla.edu
Darren Long

Department of Mathematics

University of California

Santa Barbara, CA 93106-3080

long@math.ucsb.edu

Jiang-Hua Lu

Department of Mathematics

The University of Hong Kong

Pokfulam Rd., Hong Kong jhlu@maths.hku.hk

Alexander Merkurjev

Department of Mathematics

University of California

Los Angeles, CA 90095-1555

merkurev@math.ucla.edu
Sorin Popa

Department of Mathematics University of California

Los Angeles, CA 90095-1555 popa@math.ucla.edu

Jie Qing

Department of Mathematics

University of California

Santa Cruz, CA 95064

qing@cats.ucsc.edu

Jonathan Rogawski

Department of Mathematics

University of California

Los Angeles, CA 90095-1555

jonr@math.ucla.edu

\section{PRODUCTION}

pacific@math.berkeley.edu

\section{SUPPORTING INSTITUTIONS}

ACADEMIA SINICA, TAIPEI

CALIFORNIA INST. OF TECHNOLOGY INST. DE MATEMÁTICA PURA E APLICADA KEIO UNIVERSITY

MATH. SCIENCES RESEARCH INSTITUTE NEW MEXICO STATE UNIV.

OREGON STATE UNIV.

\author{
STANFORD UNIVERSITY \\ UNIV. OF BRITISH COLUMBIA \\ UNIV. OF CALIFORNIA, BERKELEY \\ UNIV. OF CALIFORNIA, DAVIS \\ UNIV. OF CALIFORNIA, LOS ANGELES \\ UNIV. OF CALIFORNIA, RIVERSIDE \\ UNIV. OF CALIFORNIA, SAN DIEGO \\ UNIV. OF CALIF., SANTA BARBARA
}

\author{
UNIV. OF CALIF., SANTA CRUZ \\ UNIV. OF MONTANA \\ UNIV. OF OREGON \\ UNIV. OF SOUTHERN CALIFORNIA \\ UNIV. OF UTAH \\ UNIV. OF WASHINGTON \\ WASHINGTON STATE UNIVERSITY
}

These supporting institutions contribute to the cost of publication of this Journal, but they are not owners or publishers and have no responsibility for its contents or policies.

See inside back cover or pacificmath.org for submission instructions.

The subscription price for 2012 is US \$420/year for the electronic version, and \$485/year for print and electronic.

Subscriptions, requests for back issues from the last three years and changes of subscribers address should be sent to Pacific Journal of Mathematics, P.O. Box 4163, Berkeley, CA 94704-0163, U.S.A. Prior back issues are obtainable from Periodicals Service Company, 11 Main Street, Germantown, NY 12526-5635. The Pacific Journal of Mathematics is indexed by Mathematical Reviews, Zentralblatt MATH, PASCAL CNRS Index, Referativnyi Zhurnal, Current Mathematical Publications and the Science Citation Index.

The Pacific Journal of Mathematics (ISSN 0030-8730) at the University of California, c/o Department of Mathematics, 969 Evans Hall, Berkeley, CA 94720-3840, is published monthly except July and August. Periodical rate postage paid at Berkeley, CA 94704, and additional mailing offices. POSTMASTER: send address changes to Pacific Journal of Mathematics, P.O. Box 4163, Berkeley, CA 94704-0163.

PJM peer review and production are managed by EditFLOW ${ }^{\mathrm{TM}}$ from Mathematical Sciences Publishers.

PUBLISHED BY PACIFIC JOURNAL OF MATHEMATICS

at the University of California, Berkeley 94720-3840

A NON-PROFIT CORPORATION

Typeset in LATEX

Copyright $(02012$ by Pacific Journal of Mathematics 


\section{PACIFIC JOURNAL OF MATHEMATICS}

Volume $257 \quad$ No. $2 \quad$ June 2012

Extending triangulations of the 2-sphere to the 3-disk preserving a

4-coloring

RUi PEDRO CARPENTIER

Orthogonal quantum group invariants of links

LIN CHEN and QINGTAO CHEN

Some properties of squeezing functions on bounded domains

FUSHENG DENG, QIAN GUAN and LIYOU ZHANG

Representations of little $q$-Schur algebras

JiE DU, QIANG FU and JiAN-PAN WANG

Renormalized weighted volume and conformal fractional Laplacians

MARÍA DEL MAR GONZÁLEZ

The $L_{4}$ norm of Littlewood polynomials derived from the Jacobi symbol 395

JONATHAN JEDWAB and KAI-UWE SCHMIDT

On a conjecture of Kaneko and Ohno

ZHONG-HUA LI

Categories of unitary representations of Banach-Lie supergroups and restriction functors

StéPhane Merigon, Karl-Hermann NeEb and Hadi

SALMASIAN

Odd Hamiltonian superalgebras and special odd Hamiltonian superalgebras of formal vector fields

Li Ren, Qiang Mu and YongZheng ZHANG

Interior derivative estimates for the Kähler-Ricci flow

Morgan SHERMAN and BEN WEINKOVE

Two-dimensional disjoint minimal graphs

LINFENG ZHOU 